Artículo científico

(Original paper)

\title{
ARTRÓPODOS (ARTHROPODA: ARACHNIDA E INSECTA) ASOCIADOS A LOS NIDOS DE NEOTOMA MEXICANA (MAMMALIA: RODENTIA) DE SAN MIGUEL DE ALLENDE, GUANAJUATO, MÉXICO
}

\section{ARTHROPODS (ARATHROPODA: ARACHNIDA AND INSECTA) ASSOCIATED WITH THE NESTS OF NEOTOMA MEXICANA (MAMMALIA: RODENTIA) FROM SAN MIGUEL DE ALLENDE, GUANAJUATO, MEXICO}

\begin{abstract}
MARIANA S. MARTÍNEZ HERNÁNDEZ ${ }^{1}$, ZAIRA HERNÁNDEZ HERNÁNDEZ ${ }^{1}$, GABRIEL A. VILLEGAS GUZMÁN ${ }^{1,2 *}$, SALVADOR GAONA RAMÍREZ ${ }^{1}$

${ }^{1}$ Laboratorio de Conservación de Fauna Silvestre, Departamento Biología, Universidad Autónoma Metropolitana Unidad Iztapalapa, San Rafael Atlixco No. 186, Col. Vicentina, C.P. 09340, Ciudad de México, México.

<mariana.martinez.hernandez5017@gmail.com>; <sam_zaira44@yahoo.com>; <gabrvill@gmail.com>; <sgar@xanum.uam.mx> ${ }^{2}$ Laboratorio de Acarología, Departamento Zoología, Escuela Nacional de Ciencias Biológicas, Instituto Politécnico Nacional, Prolongación Carpio y Plan de Ayala s/n, Col. Casco de Santo Tomás C.P. 11340, Ciudad de México. <gabrvill@gmail.com>

* Autor para correspondencia: <gabrvill@gmail.com>
\end{abstract}

Recibido: 26/06/2019; aceptado: 13/08/2019; publicado en línea: 16/08/2019

Editor responsable: María Luisa Jiménez

Martínez-Hernández, M. S., Hernández-Hernández, Z., Villegas-Guzmán, G. A., Gaona-Ramírez, S. (2019) Artrópodos (Arthropoda: Arachnida e Insecta) asociados a los nidos de Neotoma mexicana (Mammalia: Rodentia) de San Miguel de Allende, Guanajuato, México. Acta Zoológica Mexicana (nueva serie), 35, 1-9. https://doi.org/10.21829/azm.2019.3502217

RESUMEN. Los nidos de roedores son el refugio de distintas especies de artrópodos como pueden ser: ácaros, pulgas, chinches, piojos y pseudoescorpiones, entre otros. Algunos de ellos son de importancia médica y veterinaria porque son vectores de diversas enfermedades que pueden trasmitir al ser humano, animales domésticos y silvestres. Se recolectó un nido de Neotoma a partir del cual se identificaron 148 artrópodos de tres órdenes: Acari, Pseudoscorpiones (Arachnida) (Shultz, 2007) y Siphonaptera (Insecta). La especie más abundante fue el ácaro Cheyletus eruditus (Acari: Prostigmata: Cheyletidae) con 126 ejemplares. Tectocepheus velatus (Acari: Oribatida: Tectocepheidae) es un nuevo registro para los nidos de Neotoma. Los pseudoescorpiones representan el primer registro para Guanajuato, siendo Parachernes un nuevo registro para este microhábitat. Del orden Siphonaptera Tunga monositus es un nuevo registro para Guanajuato.

Palabras clave: arácnidos; roedores; insectos; nidos

Martínez-Hernández, M. S., Hernández-Hernández, Z., Villegas-Guzmán, G. A., Gaona-Ramírez, S. (2019) Arthropods (Arathropoda: Arachnida and Insecta) associated with the nests of Neotoma mexicana (Mammalia: Rodentia) from San Miguel de Allende, Guanajuato, Mexico. Acta Zoológica Mexicana (nueva serie), 35, 1-9. https://doi.org/10.21829/azm.2019.3502217 


\begin{abstract}
The nests of rodents are the refuge of different species of arthropods such as mites, fleas, bugs, lice, and pseudoscorpions, among others. Some of them are of veterinary and medical importance because they are vectors of diverse diseases that can be transmitted to a human, domestic and wild animals. A nest of Neotoma mexicana was collected, from which 148 arthropods of four orders were found: Acari, Pseudoscorpiones (Arachnida), and Siphonaptera (Insecta). The most abundant species of mites was Cheyletus eruditus (Acari: Prostigmata: Chayletidae) with 126. Tectocepheus velatus (Acari: Oribatida: Tectocepheidae) is a new record for Neotoma nests. The pseudoscorpions represent the first records for Guanajuato, being Parachernes a new record for this microhabitat. Whilst for the order Siphonaptera Tunga monositus is a new record for Guanajuato.
\end{abstract}

Key words: arachnids; rodents; insects; nests

\title{
INTRODUCCIÓN
}

Los nidos de pequeños mamíferos constituyen una fuente importante de alimento y refugio para distintos grupos de artrópodos, como son: colémbolos, ácaros, arañas, pulgas, chinches y pseudoescorpiones. (Villegas-Guzmán \& Pérez, 2005). Algunos de ellos tienen interés médico ya que son vectores de microorganismos causantes de enfermedades, por ejemplo: las chinches pueden transmitir la enfermedad de Chagas (Peterson et al., 2002) y las garrapatas son vectores de la enfermedad de Lyme (Nicholson et al., 2009). Neotoma mexicana, Baird,1855, la rata magueyera mexicana, es una especie de roedor de la familia Cricetidae que se distribuye desde el norte de Colorado, Estados Unidos, hasta el oeste de Honduras (Cornely \& Baker, 1986), en México se encuentra en casi todo el país (León Tapia, 2014). Los nidos de $N$. mexicana se caracterizan por su gran tamaño, alcanzando los $2.5 \mathrm{~m}$ de diámetro y $1.5 \mathrm{~m}$ de alto (Rainey, 1956) y están formados generalmente por cuatro componentes: cámara de reposo (CR), cámara verde (CV), pasajes (PA) y cobertura (CO) (Fig. 1) (Villegas-Guzmán \& Polaco, 2006). Los materiales que usan para la construcción son acarreados de los alrededores del nido aproximadamente de 30-50 metros alrededor de su madriguera (Vaughan, 1990), entre los que se encuentran: excretas de ganado vacuno y equino, ramas de diferentes arbustos y árboles, restos de cactáceas, tunas, entre otros. En relación a la estructura de los nidos de $N$. mexicana se conocen dos estudios de Durango, en uno de ellos se recolectaron los nidos en un bosque de pino-encino en el municipio de Santiago Papasquiaro, Durango, donde los nidos se encontraban debajo de rocas, la CO estaban formada por palos, estróbilos de pino y hongos, en la CV había semillas de plantas, hongos, huesos de otros animales, piñones, hojas y tallos frescos; la CR están formados por pasto seco, papel o tela deshilachada (Villegas-Guzmán \& Polaco, 2006). En el otro estudio realizado en La Michilía, Durango, los nidos se recolectaron en dos hábitats en un bosque de pino-encino y en terreno rocoso. En estos nidos la $\mathrm{CO}$ estaba formada por ramas secas de diferentes tamaños, trozos de madera y corteza, huesos, estróbilos de pinos, piedras y excrementos de diferentes animales, en la CV se encontraban vegetales secos, hongos, pteridofitas, briofitas y líquenes; La CR estaba formada por pasto seco, plumas y pelos de la propia rata (Álvarez et al., 1988).

Los artrópodos registrados en nidos de N. mexicana, son escasos, sin embargo, en un trabajo reportan artrópodos clasificados en tres clases y 13 órdenes: 1): clase Arachnida: Araneae, Pseudoscorpiones y Acari, de este último orden estuvieron representados cinco grupos principales de ácaros (Ixodida, Mesostigmata, Prostigmata, Astigmatina y Oribatida); 2) clase Insecta: Psocoptera, Collembola, Dyctyoptera, Hemiptera, Thysanopetra, Coleoptera, Lepidoptera, Diptera, Siphonaptera e Hymenoptera; y 3) clase Diplopoda (Villegas-Guzmán et al., 1998). En otros estudios de los nidos de N. mexicana se han registrado tres especies de ácaros mesostigmados: Sertitympanum mexicanum, Kleemannia plumigera, Ameroseius sp. (Villegas-Guzmán et al., 2004), así como cinco especies de pseudoescorpiones: Juxtachelifer fructuosus (Cheliferidae), Paraliochthonius sp. (Chthoniidae), Larca chamberlini (Larcidae), Tychochernes inflatus e Illinichernes distinctus (Chernetidae) (Villegas-Guzmán \& Pérez, 2005). 
La manera en que $N$. mexicana construye sus nidos y los materiales que usa generan un ambiente propicio para el desarrollo de diversos grupos de artrópodos. El objetivo del presente trabajo fue identificar taxonómicamente las especies de artrópodos asociadas a un nido de $N$. mexicana. Así como analizar su distribución en sus componentes de este.

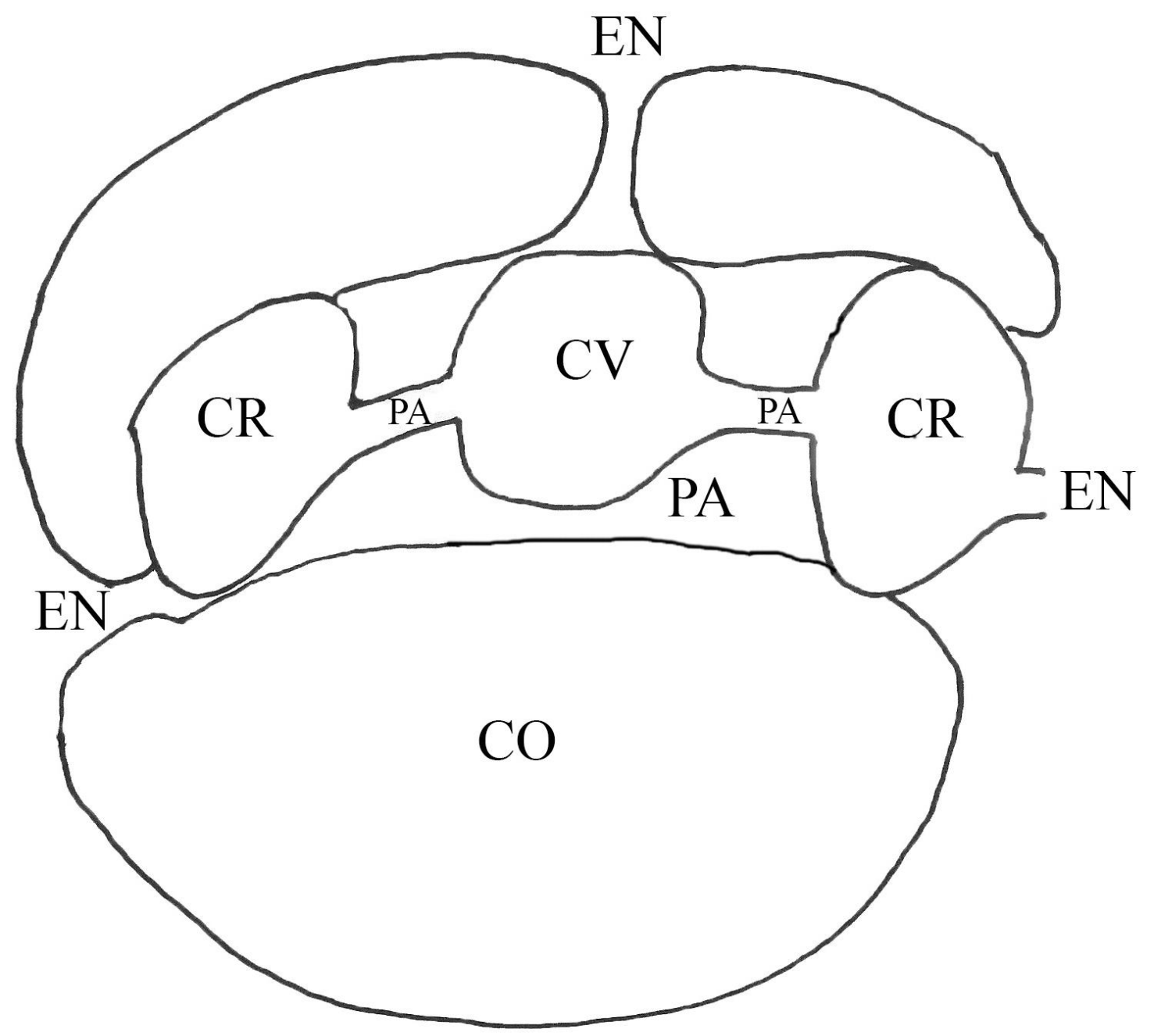

Figura 1. Esquema de un nido de Neotoma de Durango. Tomado y modificado de (Montiel-Parra et al., 2001) Abreviaturas: $\mathrm{CV}=$ cámara verde, $\mathrm{CR}=$ cámara de reposo, $\mathrm{CO}=$ cobertura, $\mathrm{PA}=$ pasaje, $\mathrm{EN}=$ entrada.

\section{MATERIALES Y MÉTODOS}

El área de estudio se ubica en el "Rancho El Estribo" a la altura del km 11+400 de la carretera nueva a Guanajuato $\left(20^{\circ} 56^{\prime} 28.8^{\prime \prime} \mathrm{N}, 100^{\circ} 52^{\prime} 23.4^{\prime}\right.$ ” W, $\left.1878 \mathrm{msnm}\right)$. El tipo de vegetación corresponde a matorral xerófilo, el cual se encuentra muy perturbado. El nido fue encontrado a nivel el suelo en la base de una nopalera (Fig. 2) y se desmanteló el 22 de febrero del 2018, separando componentes: la cobertura, la cámara verde y la cámara de reposo. El material del nido se procesó con la técnica del embudo de Berlese-Tullgren para obtener los artrópodos asociados (Márquez-Luna, 2005). Los ácaros se transparentaron con lactofenol y se montaron entre porta y cubre objetos con medio de líquido de Hoyer. Los pseudoescorpiones y pulgas se procesaron mediante la técnica de Wirth \& Marston (1968). Una vez montados todos los organismos, las 
preparaciones se colocaron en la estufa durante 15 días para su secado, posteriormente las preparaciones de ácaros se sellaron con pintura alquílica para proceder a su identificación (Acosta \& Morrone, 2003; Baker, 1949; Muchmore, 1990; Muchmore \& Alteri, 1974). Los organismos están depositados en la Colección de Artrópodos Asociados a Mamíferos Silvestres de la Universidad Autónoma Metropolitana Iztapalapa (CAAMS-1-153).

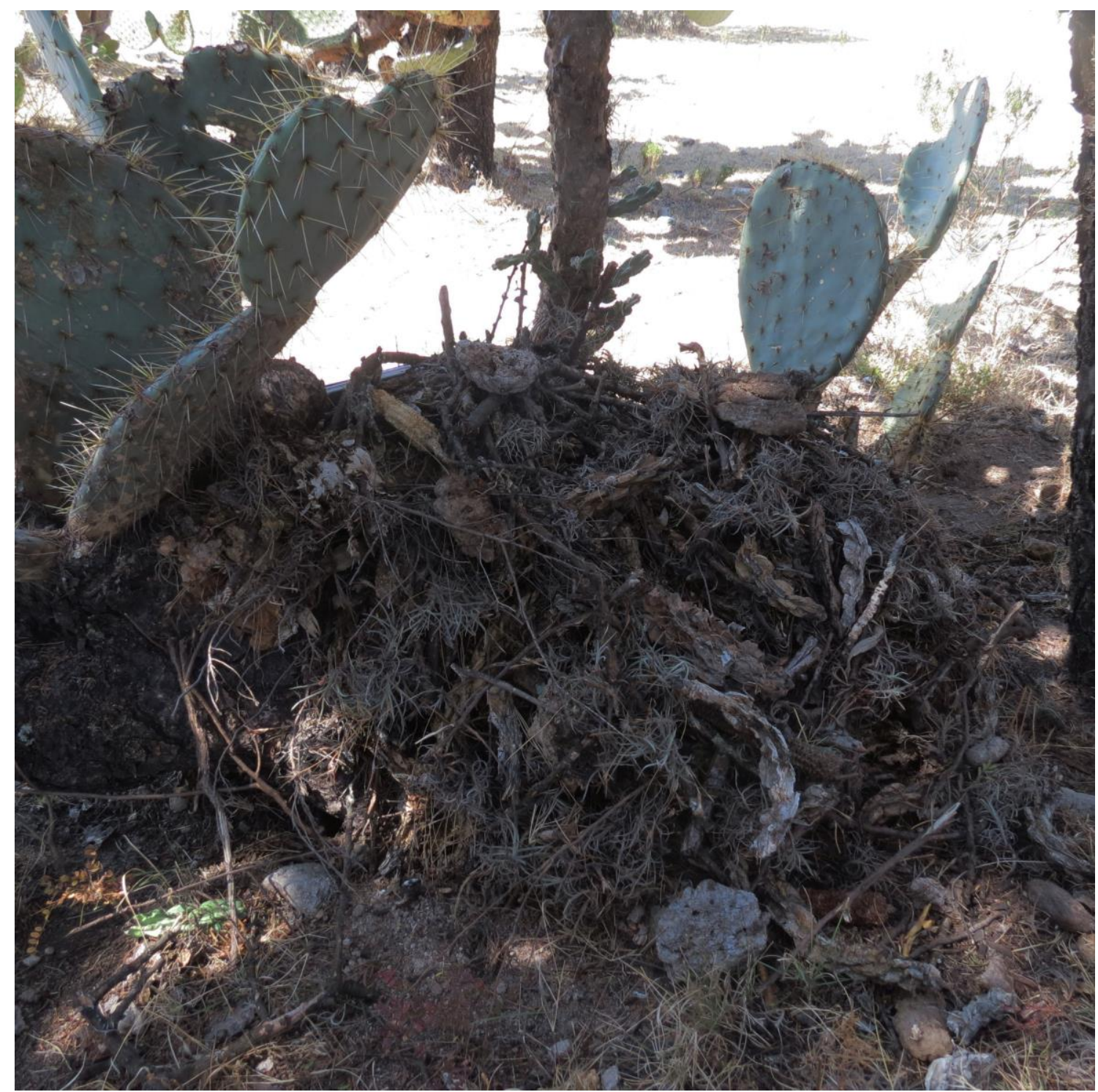

Figura 2. Nido de Neotoma mexicana del rancho "El Estribo", San Miguel de Allende, Guanajuato.

\section{RESULTADOS}

Se recuperaron 148 artrópodos de dos clases (Arachnida e Insecta) y tres órdenes: Acari, Pseudoscorpiones y Siphonaptera. En el nido se observaron tres componentes: cobertura, cámara de reposo y cámara verde, no se encontraron a los pasajes, sin embargo, en la cobertura se apreciaron diferentes entradas, por lo que probablemente eran pasajes que al momento de ir retirando los componentes de la cobertura como eran: nopales, palos, excretas de ganado, piedras, entre otros, se perdieron. Los artrópodos se encontraron en dos componentes: Cámara verde (114) y Cámara de reposo (34). La cámara verde, estaba formada por restos de 
plantas (nopales y cardón), frutos (tunas), ramas secas y excretas de Neotoma. Los ácaros identificados pertenecen a dos especies: Cheyletus eruditus con 113 organismos y una hembra Chortoglyphus arcuatus (Cuadro 1). La cámara de reposo estuvo formada por pasto seco y restos de tela. En este componente se obtuvieron seis especies: cuatro de ácaros; Androlaelaps geomys (14 organismos), Cheyletus eruditus (7), Cheletoides sp. (1) y Tectocepheus velatus (1); tres pseudoescorpiones identificados como Parachernes ca robustus. y nueve pulgas Tunga aff. monositus (Cuadro 1).

Cuadro1. Especies de artrópodos por sitios encontradas en el nido de Neotoma mexicana de San Miguel de Allende, Guanajuato. Abreviaturas: $\mathrm{CV}=$ cámara verde, $\mathrm{CR}=$ cámara de reposo, $\mathrm{D}=$ deutoninfa.

*nuevos registros en nidos de N. mexicana

\begin{tabular}{|c|c|c|c|}
\hline Orden/Familia & Especie & Ejemplares & Componente \\
\hline $\begin{array}{c}\text { Arachnida } \\
\text { Acari/ }\end{array}$ & & & \\
\hline Cheyletidae & Cheyletus eruditus & 33 오 y 86 ठ & $\mathrm{CV}$ \\
\hline & Cheyletus eruditus & $70^{\pi}$ & $\mathrm{CR}$ \\
\hline & Cheletoides sp. & & $\mathrm{CR}$ \\
\hline Chortoglyphidae & Chortoglyphus arcuatus & & $\mathrm{CV}$ \\
\hline Laelapidae & Androlaelaps geomys & 11 甲 у 3 ठ & $\mathrm{CR}^{*}$ \\
\hline Tectocepheidae & Tectocepheus velatus & 1 우 & $\mathrm{CR}^{*}$ \\
\hline Pseudoscorpiones & & & \\
\hline Chernetidae & Parachernes ca robustus & $1 \circ$ y $2 \mathrm{D}$ & $\mathrm{CR}^{*}$ \\
\hline $\begin{array}{c}\text { Insecta } \\
\text { Siphonaptera/ } \\
\text { Tungidae }\end{array}$ & Tunga aff. monositus & $4 \hat{\circ}$ y 5 ㅇ & $\mathrm{CR}$ \\
\hline
\end{tabular}

\section{DISCUSIÓN}

Los nidos de $N$. mexicana presentan condiciones ambientales con una alta humedad y una temperatura moderada comparada con el exterior (Drummond, 1957) convirtiendo a los nidos en microhábitats con las condiciones adecuadas para el desarrollo de diferentes artrópodos (Villegas-Guzmán \& Pérez, 2005), por ello en este trabajo encontramos organismos de dos clases (Arachnida e Insecta) y tres órdenes (Acari, Pseudoscorpiones y Siphonaptera) debido a que aquí encuentran las condiciones físicas adecuadas para su desarrollo, así como el alimento que requieren para ello. Esto coincide con lo registrado en un nido de $N$. mexicana en Durango (Villegas-Guzmán et al., 1998). Sin embargo, en el nido de Durango se encontró una mayor riqueza y abundancia, esto probablemente se deba al tipo de hábitat y a la época del año donde fue recolectado, ya que se encontró en un bosque de pino-encino y en época lluviosa (agosto de 1996) donde hay una mayor cantidad de recursos que puede ayudar a la proliferación de lo artrópodos, mientras el nido de Guanajuato se recolectó en un matorral xerófilo y en época de estiaje (febrero de 2018). Otra probable explicación de esto es que este nido había sido recientemente abandonado, razón por la cual se encontraron pocos organismos especialmente hematófagos en la cámara de reposo, en este componente en el nido de Durango se encontraron pulgas, argásidos, laelápidos y macronísidos de forma abundante (VillegasGuzmán et al., 1998), y no había artrópodos en la cobertura del nido ya que no había materiales frescos en este componente.

En trabajos previos se ha reportado que los ácaros son los más abundantes, como se observa en este estudio donde representan más del 96\%. Familias como Cheyletidae y Laelapidae han sido reportadas para nidos del género Neotoma (Allred \& Roscoe, 1957; Cudmore, 1986). Montiel-Parra et al. (2001) registra 110 ejemplares de Laelapidae y 67 de Cheyletidae de un nido de N. albigula. Estos resultados coinciden parcialmente con los observados en este trabajo. 
En la cámara verde se encontró la mayor cantidad de ejemplares (114) de dos especies del orden Acari (Cuadro 1). El artrópodo más abundante del nido fue Cheyletus eruditus que se observó en ambas cámaras (CR y CV), presentando en la cámara verde su mayor abundancia, esto se debe a que este ácaro es habitante común en granos almacenados donde se alimenta de ácaros fitófagos, psocópteros, larvas de polillas y coleópteros, e incluso se ha observado que son caníbales (Coombs \& Woodroffe, 1968; Hughes, 1976). El otro habitante de la cámara verde es Chortoglyphus arcuatus de quien solo se encontró una hembra, esta especie se ha registrado en graneros, establos, granjas, productos almacenados y asociado a nidos de gorrión (Hughes, 1976; McDaniel, 1979), esta especie se alimenta de los restos de plantas y semillas que son llevados por el roedor.

En la cámara de reposo solo se encontraron 34 organismos, sin embargo, aquí se observó la mayor riqueza con seis especies (Cuadro 1). Los organismos encontrados generalmente son parásitos del roedor como es el caso de los laelápidos y sifonápteros, mientras que los pseudoescorpiones son foréticos y usan al roedor para desplazarse (Villegas-Guzmán \& Hernández-Betancurt, 2006). Se han reportado a 32 especies de pseudoescorpiones asociadas a nidos de Neotoma (Francke \& Villegas-Guzmán, 2006), en los cuales pueden estar presentes todos los estadios de su ciclo de vida, por lo cual se encuentran adultos y estadios ninfales como en nuestro caso. En N. mexicana se han registrado cinco especies (Villegas-Guzmán \& Pérez, 2005), dos de ellas de la familia Chernetidae, Illinichernes distinctus y Tychochernes inflatus, por lo cual es la primera vez que se registra a Parachernes asociado a nidos de esta especie de roedor. Los pseudoescorpiones representan los primeros registros para Guanajuato, siendo Parachernes un nuevo registro para este microhábitat, ya que solo se había encontrado asociado a cortezas de árbol en Chiapas (Córdova-Tabares \& Villegas-Guzmán, 2013), es probable que este organismo haya sido acarreado dentro de los materiales que la rata llevó para formar su nido, por lo que consideramos que no es raro que se haya encontrado en el mismo.

Los laelápidos Androlaelaps geomys en México se han registrado sobre la tuza (Pappogeomys) (Bassols, 1981), también se ha registrado un solo ejemplar sobre Peromyscus y Neotoma, aunque Strandtmann (1949) los considera accidentales. El hecho de encontrar a A. geomys en el nido de N. mexicana representa el primer registro de en este microhábitat. Existen diez especies de Laelapidae asociadas a seis especies de Neotoma (Allred \& Beck, 1966; Keegan, 1949; Henrrin 1970). De Androlaelaps se han registrado sobre varias especies de Neotoma, por ejemplo, a A. fahrenholzi (Berlsese) se ha encontrado sobre N. microspus, N. cinerea y N. pennsylvanica (Strandtmann, 1949). Mientras que, a A. circularis (Ewing) se encuentra asociada sobre N. mexicana en Estados Unidos (Allred \& Beck, 1966; Whitaker \& Wilson 1974). La presencia de $A$. geomys en los nidos posiblemente no sea accidental, ya que si bien no son muy numerosos (14 ejemplares), si están presentes ambos sexos, siendo las hembras más abundantes lo cual coincide con lo dicho por Strandtmann (1949) quien menciona que este sexo en los ácaros parásitos es más prolífico, además indica que los machos y ninfas están presentes en los nidos, como se observa en este estudio.

Las especies Tectocepheus velatus y Cheletoides sp., son nuevos registros para los nidos de Neotoma. T. velatus es una especie cosmopolita que se ha reportado anteriormente en el suelo de hábitats perturbados (Norton \& Behan-Pelletier, 2009) y en hormigueros (Peralta \& Martínez, 2013), el nido estudiado se encontraba en la base de una nopalera y al ras del suelo, por lo que posiblemente al recolectar los materiales de la CR se tomó suelo y accidentalmente al ácaro. Cabe resaltar que los ejemplares identificados son de mayor tamaño a los reportados anteriormente en la literatura, sin embargo, coinciden con todas las características de la especie (Caballero \& Iturrondobeitia, 2000).

\section{CONCLUSIONES}

La distribución de los artrópodos en los componentes de los nidos se debe a los recursos que ofrece cada uno de ellos, al ambiente donde se localizan y a los hábitos de los organismos. En el caso de la cámara verde 
generalmente vamos a encontrar organismos fitófagos y depredadores, mientras que en la cámara de reposo se van a encontrar organismos hematófagos, depredadores y foréticos. Consideramos que es propicio seguir realizando este tipo de estudios para conocer que artrópodos habitan en los nidos y sus relaciones tróficas entre ellos, sobre todo en aquellas zonas del país donde sus registros son escasos; como es el caso de Guanajuato, por lo que los resultados observados en este trabajo son nuevos registros para el estado.

AgRAdECIMIENTOS. Los autores agradecen a Vicente Yazbec dueño del rancho "el Estribo" por permitirnos recolectar el nido en su propiedad. A Marlene Méndez y Jesús Campos por su colaboración en el trabajo de campo. A PRODEP por el apoyo al proyecto: "Ectoparásitos asociados a roedores de las familias Cricetidae y Heteromyidae del municipio de San Miguel de Allende, Guanajuato". A Ricardo Paredes-León y a dos revisores anónimos por sus comentarios y sugerencias que enriquecieron al manuscrito.

\section{LITERATURA CITADA}

Acosta, R., Morrone, J. J. (2003) Clave ilustrada para la identificación de los taxones supraespecíficos de Siphonaptera de México. Acta Zoológica Mexicana, 89, 39-53.

Allred, D. M., Beck, D. E. (1966) Mites of Utah mammals. Brigham Young University Science Bulletin, Biological Series, 8, 1-123.

Allred, D., Roscoe, E. (1957) Parasitic Mites in Desert Wood Rat Nests with Notes on Free-Living Forms. Transactions of the American Microscopical Society, 76, 389-403.

Álvarez, T., López-Vidal, J. C., Polaco, O. J. (1988) Estudio de las madrigueras de la rata magueyera Neotoma mexicana (Rodentia), en la reserva de la biósfera La Michilía, Durango, México. Anales de la Escuela Nacional de Ciencias Biológicas, México, 32, 131-154.

Baker, E. W. (1949) A review of the mites of the family Cheyletidae in the United States National Museum. Proceedings of the United States National Museum, 99, 267-320.

Bassols, I. B. (1981) Catálogo de los ácaros Mesostigmata de mamíferos de México. Anales de la Escuela Nacional de Ciencias Biología, México, 24, 9-49.

Caballero, A. I., Iturrondobeitia, J. C. (2000) Aportaciones al conocimiento de la especie Tectocepheus velatus (Michael, 1880) (Acari: Oribatida: Tectocepheidae). Boletín de la Asociación española de Entomología, 24, 9-13.

Coombs, C. W., Woodroffe, G. E. (1968) Changes in the arthropod fauna of an experimental bulk of stored wheat. Journal of Applied Ecology, 5, 563-574.

Córdova-Tabares, V. M., Villegas-Guzmán, G. A. (2013) Nuevos registros de pseudoescorpiones (Arachnida: Pseudoscorpiones) en Chiapas, México. Acta Zoológica Mexicana, 29, 596-613.

Cornely, J. E., Baker R. J. (1986) Neotoma mexicana. Mammalian species, 262, 1-7.

Cudmore, W.W. (1986) Nest associates and ectoparasites of the eastern wood rat, Neotoma floridana, in Indiana. Canadian Journal of Zoology, 64, 353-357.

Drummond, O. R. (1957) Observations on fluctuations of acarine populations from nests of Peromyscus leucopus. Ecological Monographs, 27, 13-152.

Francke, O. F., Villegas-Guzman, G. A. (2006) Symbiotic relationships between pseudoscorpions (Arachnida) and packrats (Rodentia). Journal of Arachnology, 34, 289-298.

Henrrin, C. S. (1970) A systematic revision of the genus Hirstionyssus (Acari: Mesostigma) of the Nearctic region. Journal of Medical Entomology, 7, 391-437.

Hughes, A. M. (1976) The mites of stored and houses. Ministry of agriculture, fisheries and Food. London.

Keegan, H. L. (1949) Euhaemogamasus utahensis Ewing, 1933 Redescribe as type species of new genus Brevisterna (Acarina: Laelaptidae). Transactions of the American Microscopical Society, 48, 222227. 
León Tapia, M. A. (2014) Los roedores múridos de México: los pequeños mamíferos exitosos. CONABIO. Biodiversitas, 113, 8-11.

Márquez-Luna, J. (2005) Técnica de colecta y preservación de insectos. Boletín Sociedad Entomológica Aragonesa, 37, 385-408.

McDaniel, B. (1979) How to know the mites and ticks. The pictured key nature serie. Brown Company Publishers, Iowa.

Montiel-Parra, G, Villegas-Guzmán, G. A., Vargas, M., Polaco, O. J. (2001) Mites associated with nests of Neotoma albigula Hartley, 1894 (Rodentia: Muridae) de Durango, México, pp. 586-593. In: Halliday, R. B., Walter, D. E., Proctor, H. C., Norton, R. A., Colloff, M. J. (Eds.). Acarology: Proceedings of the 10th International Congress. CSIRO Publishing, Melbourne.

Muchmore, W. B. (1990) Pseudoscorpionida, pp. 503-527. In Dindal, D. L. (ed). Soil biology guide. John Wiley and Sons. New York.

Muchmore, W. B., Alteri, C. (1974) The genus Parachernes (Pseudoscorpionida, Chernetidae) in the United States, with description of new species. Transactions of the American Entomologist Society, 99, 477-506.

Nicholson, W. L., Sonenshine, D. E., Lane, R. S., Uilenberg, G. (2009) Ticks (Ixodida), pp. 493-542. In: Mullen, G. R., Durden, L. A. (Eds.). Medical and Veterinary Entomology. Second Edition. Academic Press, San Diego.

Norton, R. A., Behan-Pelletier, V. M. (2009) Surborden Oribatida, pp. 430-564. In: Krantz, G. W., Walter, D. E. (Eds.) A manual of Acarology. Third Edition. Texas Tech Press, USA.

Peralta, L., Martínez, P. A. (2013) Ensambles de ácaros oribátidos en hormigueros de Acromyrmex spp. (Hymenoptera, Formicidae). Ecología austral, 23, 209-217.

Peterson, A. T., Sánchez-Cordero, V., Beard, B. C., Ramsey, J. M. (2002) Ecologic Niche Modeling and Potential Reservoirs for Chagas Disease, Mexico. Emerging Infectious Disease, 8, 662-667. https://dx.doi.org/10.3201/eid0807.010454

Rainey, D. G. (1956 Eastern woodrat, Neotoma floridana: life history and ecology. University of Kansas Publications Museum of Natural History, 8, 535-646.

Shultz, J. W. (2007) A phylogenetic analysis of the arachnid orders based on morphological characters. Zoological Journal of the Linnean Society, 150, 221-265.

Strandtmann, R. W. (1949) The blood-sucking mites of the genus Haemolaelaps (Acari: Laelaptidae) in the United States. The Journal of Parasitology, 35, 325-352.

Vaughan, T. A. (1990) Ecology of living packrats, pp. 14-27. In: Betancourt, J. L., Van Devender, T. R., Martin P. S. (Eds.). Packrat Middens, The last 40,000 years of biotic Change. The University of Arizona Press, Tucson.

Villegas-Guzmán, G. A., Hernández-Betancourt, S. (2006) Pseudoescorpiones foréticos de roedores en México. Acta Zoológica Mexicana (nueva serie), 22, 141-143.

Villegas-Guzmán G. A., Pérez, T. M. (2005) Pseudoescorpiones (Arachnida: Pseudoscorpionida) asociados a nidos de ratas del género Neotoma (Mammalia: Rodentia) del Altiplano Mexicano. Acta Zoológica Mexicana (nueva serie), 21, 63-77.

Villegas-Guzmán, G. A., Polaco, O. J. (2006) Estructura de las madrigueras de cinco especies del género Neotoma del Altiplano Mexicano. Vertebrata Mexicana, 18, $23-28$.

Villegas-Guzmán, G. A., Montiel-Parra, G., Vargas, M., Polaco, O. J. (1998) Los artrópodos habitantes de nidos de Neotoma mexicana Baird, 1855 (Rodentia: Muridae), pp. 91-96. Memorias del XXXIII Congreso Nacional de Entomología, Guerrero, México.

Villegas-Guzmán, G. A., Montiel-Parra, G., Vargas, M., Polaco, O. J. (2004) Ameroséidos asociados a nidos de Neotoma mexicana Baird, 1855 (Rodentia: Muridae) y descripción de una nueva especies del género Sertitympanum Elsen y Whithanker, 1985. Acta Zoológica Mexicana (nueva serie), 20, 27-36.

Whitaker, J. O. Jr., Wilson, N. (1974) Host and distribution lists of mites (Acari), parasitic and phoretic, in the hair of wild mammals of North America, North of Mexico. The American Midland Naturalist, $91,1-67$. 
Wirth, W.W., Marston, N. (1968) A method for mounting small insects on microscope slides in Canada balsam. Annals of the Entomological Society of America, 61, 783-784. 